\title{
BAYESIAN ANALYSIS OF INFANT'S GROWTH DYNAMICS WITH IN UTERO EXPOSURE TO ENVIRONMENTAL TOXICANTS ${ }^{1}$
}

\author{
By Jonggyu Baek, Bin Zhu And Peter X. K. Song \\ University of Michigan, University of Massachusetts Medical School and \\ National Institutes of Health
}

\begin{abstract}
Early infancy from at-birth to 3 years is critical for cognitive, emotional and social development of infants. During this period, infant's developmental tempo and outcomes are potentially impacted by in utero exposure to endocrine disrupting compounds (EDCs), such as bisphenol A (BPA) and phthalates. We investigate effects of ten ubiquitous EDCs on the infant growth dynamics of body mass index (BMI) in a birth cohort study. Modeling growth acceleration is proposed to understand the "force of growth" through a class of semiparametric stochastic velocity models. The great flexibility of such a dynamic model enables us to capture subject-specific dynamics of growth trajectories and to assess effects of the EDCs on potential delay of growth. We adopted a Bayesian method with the Ornstein-Uhlenbeck process as the prior for the growth rate function, in which the World Health Organization global infant's growth curves were integrated into our analysis. We found that BPA and most of phthalates exposed during the first trimester of pregnancy were inversely associated with BMI growth acceleration, resulting in a delayed achievement of infant BMI peak. Such early growth deficiency has been reported as a profound impact on health outcomes in puberty (e.g., timing of sexual maturation) and adulthood.
\end{abstract}

1. Introduction. The Developmental Origin of Health and Disease (DOHaD) hypothesis is an essential theoretical framework in environmental health sciences. One of its central aims is to study how prenatal and early postnatal risk factors influence children's growth and developmental outcomes. Environmental endocrine disrupting compounds (EDCs) such as bisphenol A (BPA) and phthalates are examples that have long-term detrimental effects on human growth. BPA is a high production chemical used in the manufacture of polycarbonate plastics, epoxy resins and thermal paper, while phthalates are a diverse class of high-production industrial chemicals used to make plastics more flexible. Both BPA and phthalates are widely in use, and the majority of women are constantly exposed [Nhanes (2009)]. These EDCs affect both lipid metabolism and regularization of hormones [Casals-Casas, Feige and Desvergne (2008)] and possibly alter the growth and development trajectory of children [Diamanti-Kandarakis et al. (2009)].

Received July 2017; revised May 2018.

${ }^{1}$ Supported by National Institute of Environmental Health Sciences Grant R01ES024732.

Key words and phrases. Body mass index, Markov chain Monte Carlo (MCMC), OrnsteinUhlenbeck process, prenatal exposure, semiparametric stochastic velocity model. 


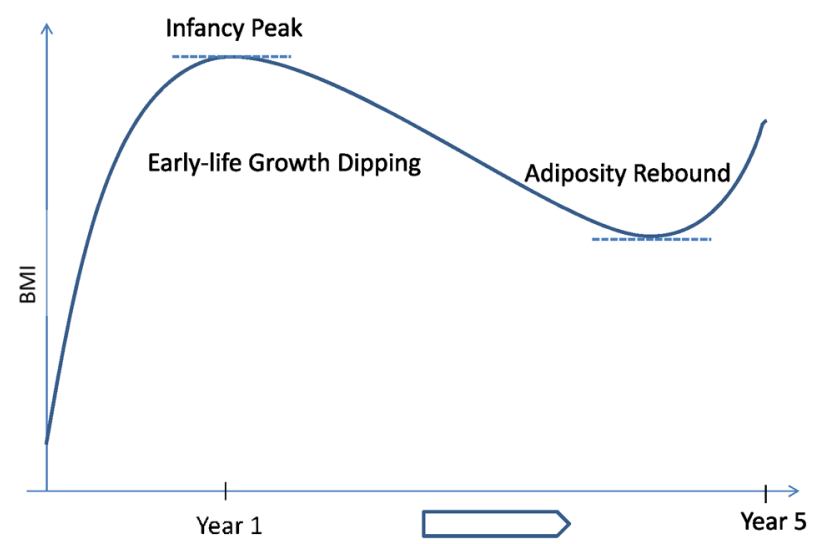

FIG. 1. Conceptual population-level child growth BMI pattern for children aged 0 to 5 years according to the World Health Organization.

Infancy is of the greatest importance not only for the cognitive, emotional and social development [Piaget (2000), Saarni (1999)], but also for the physical changes of transitioning from an infant to a toddler. In effect, this period is dominated by two major growth processes: the faster growth of weight than height over the first six months or so after birth, followed by the faster growth of height than weight. This height-weight growth pace yields the first human growth milestone, the infant body mass index (BMI) peak (see Figure 1). The infant BMI peak occurs on average at age one or so and has been found to be associated with BMI in later childhood [Silverwood et al. (2005)], which consequently has a profound impact on infant's putative later outcomes (e.g., sexual maturation and obesity) [Jensen et al. (2015), Jones-Smith et al. (2013), Taylor et al. (2005)]. Hence, studying associations of the child's growth modes such as the infant BMI peak and adiposity rebound with environmental exposures is of great importance in the life-course epidemiology to address the DOHaD hypothesis.

Statistical contributions to the methodology for analyzing growth curves have been abundant, including both parametric and nonparametric approaches [Botton et al. (2008), Cole, Donaldson and Ben-shlomo (2010), Jenns and Bayley (1937), López-Pintado and McKeague (2013), McKeague et al. (2011), Preece and Baines (1978)]. The existing methods have often focused on modeling growth mean trajectory and velocity, such as SuperImposition by Translation And Rotation (SITAR) approach recently developed by Cole, Donaldson and Ben-shlomo (2010). SITAR directly models a growth outcome via a nonlinear function within a generalized linear mixed framework in which subject-specific "shift" and "scale" random effects are used to characterize growth size, timing of peak velocity and velocity. The subject-specific parameters in a SITAR model for velocity are useful to perform an association analysis with exposures.

We adopt a dynamic model based on a stochastic differential equation to investigate infant's growth pattern and potential growth changes associated with environ- 
mental exposure to EDCs. Our model formulation is originated from the Newton's laws of motion because acceleration, not velocity, drives motion. There have been no studies concerning growth acceleration modeling, in spite of the fact that acceleration is a more fundamental characterization of dynamics than velocity.

The goal of this paper is to jointly analyze three elements of motion: BMI growth trajectory, velocity and acceleration, which are referred to as growth dynamics in the rest of this paper. To incorporate the existing World Health Organization (WHO) population growth data into our analysis, we adopt a Bayesian method. Our analysis objective is to investigate whether, and how, in utero exposure to each, or a mixture, of EDCs in pregnant women during three trimesters is associated with infant BMI growth dynamics. Specifically, due to distinctive developmental phases every 3 months of pregnancy, we investigate associations of each trimester exposure to EDCs with infant BMI growth, separately.

Several statistical challenges arise in the association analysis using continuoustime dynamic models. First, the number of longitudinal visits is relatively small with fixed schedules (i.e., 8 time points of at-birth, 3, 6, 12, 18, 24, 30, 36 months). At such paneled longitudinal visits, limited amounts of between-visit sample variabilities are available for growth curve estimation. Given the fact that human anthropometric growth is a highly regulated process, we postulate some regularity conditions to alleviate the limitation of such sparse data via the following strategies: (i) restrain short-scale growth dynamics over the time periods where no data are recorded, and (ii) optimize large-sale growth dynamics by utilizing auxiliary WHO data. Through these strategies, we are able to develop a flexible model that can not only effectively capture individual-level BMI growth dynamics, but also relate these dynamics with exposure to EDCs to address the DOHaD hypothesis.

In this paper, we extended a Bayesian semiparametric stochastic velocity model (B-SSVM) [Zhu, Taylor and Song (2011)] to formulate infant BMI growth dynamics. One of our new contributions to growth curve modeling is an important extension of the B-SSVM, which allows us to examine how prenatal exposure to EDCs is associated with postnatal growth acceleration of BMI. Our approach differs from the previous application of the B-SSVM; we focus on inference, such as testing for association of BMI growth acceleration with EDC exposure, instead of focusing on prediction as in the previous implementation. We further improve some aspects over the previous B-SSVM implementation; (i) we incorporate relevant auxiliary data (e.g., WHO data) to improve growth curve estimation; (ii) we implement a more efficient MCMC method via a direct posterior sampling that evaluates the exact transition density function of B-SSVM. This improved computational efficiency is crucial to make the proposed approach practically useful.

The remainder of the article is organized as follows. Section 2 describes our motivating data from the Early Life Exposures in Mexico to ENvironmental Toxicants (ELEMENT) study. Section 3 presents the formulation of our proposed growth dynamic model. Section 4 illustrates application of the proposed method to the ELEMENT data analysis. Section 5 reports simulation results for an experiment 
examining roles of the model parameters influencing the estimated growth dynamics.

2. ELEMENT longitudinal cohort data. The ELEMENT cohort includes three birth cohorts (cohort 1 in 1994-1997, cohort 2 in 1997-2000, cohort 3 in 2001-2005) from Mexico City maternity hospitals. Mother-infant pairs are followed for over two decades to learn how environmental exposures to metals and chemicals affect pregnant women and children.

Study population. The study population includes 250 pregnant women from a subset of the ELEMENT birth cohorts (cohort 2 and 3) [González-Cossío et al. (1997), Téllez-Rojo et al. (2004), Zhang et al. (2012)]. These mothers were followed during pre/post-natal periods, completing interview-administered questionnaires and anthropometry evaluations, and provided biological samples for EDC analysis. We excluded infants with missing in utero exposure data $(n=21)$ or premature birth that is less than 37 weeks of gestation $(n=16)$; this exclusion criterion has been suggested to reduce bias for postnatal catchup growth [Afeiche et al. (2011), Binkin et al. (1988)]. In addition, using BMI z-score (BMI-z) calculated on the reference of the WHO growth chart [WHO (2016)], we further excluded biologically implausible values of $|\mathrm{BMI}-\mathrm{z}|>5$. The resulting numbers of infants with complete observations in our analysis are $n=185, n=184$ and $n=180$ (and 1257,1269 and 1239 repeated measures) over three trimesters, respectively.

Outcome. The outcome of interest is infant BMI [weight $(\mathrm{kg}) /$ length $\left(\mathrm{m}^{2}\right)$ ], where weight and length were measured longitudinally at eight times, namely atbirth, 3, 6, 12, 18, 24, 36 months. Due to slightly different observational times across infants, clinicians have clustered them as 8 fixed times in the data reporting. Figure 2 displays some summary statistics regarding the data collection scheme used in the ELEMENT cohort study.

Environmental exposure. BPA and phthalates are the EDC exposure of interest in our analysis to address the DOHaD hypothesis. Specifically, our scientific ob-

(a)

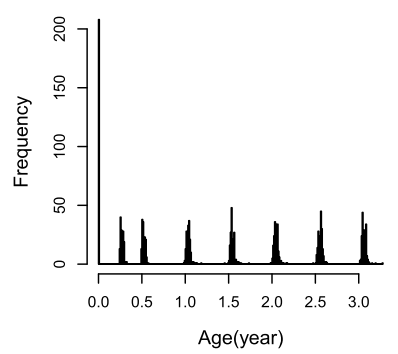

(b)

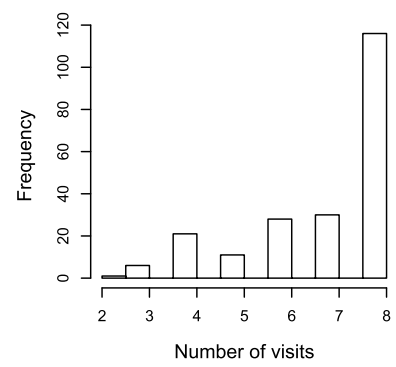

(c)

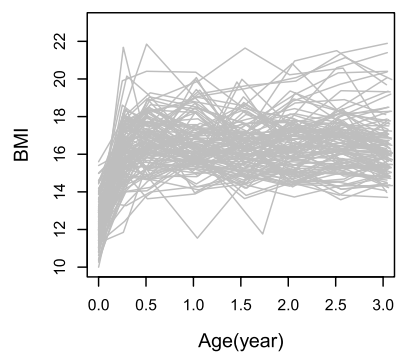

FIG. 2. (a) Number of visits at 8 fixed visit times from at-birth to 3 years. (b) Total number of visits across infants. (c) Longitudinal infant BMI trajectories among 116 infants with complete BMI measured at all 8 visits. Enlarged figures are available in the Supplementary Material [Baek, Zhu and Song (2019)]. 
jective is to examine the association of growth dynamics with exposure to EDCs. BPA and nine phthalates were measured from urine samples of mothers during three trimesters of pregnancy by isotope dilution-liquid chromatography-tandem mass spectrometry (ID-LC-MS/MS) at Lab NSF International (Ann Arbor, MI). Nine phthalates include MEHP, MEHHP, MEOHP, MECPP, MBzP, MBP, MiBP, MEP and MCPP. Full names of the nine phthalates and the procedure to calculate specific-gravity adjusted EDCs are available in the Supplementary Material [Baek, Zhu and Song (2019)]. In the analysis, we used log-transformed specific-gravity adjusted EDCs.

Given the fact that prenatal exposure occurs in forms of mixtures, or combinations of phthalates, it is of interest to evaluate the association of EDC mixtures in addition to a single EDC with growth dynamics. We considered the following mixtures of great popularity: (i) SumPCP: sum of MBP and MEP, which is regarded as an estimate of phthalate exposure from shampoos, conditioners, perfumes, nail polishes and other personal care products [Braun et al. (2014), Kobrosly et al. (2012), Schettler (2006)]; (ii) SumDEHP: sum of MECPP, MEOHP, MEHHP and MEHP, which gives an estimate of DEHP exposure from products such as PVC plastics used in food processing/packaging materials as well as building materials and medical devices [Braun et al. (2014), Kobrosly et al. (2012), Schettler (2006)]; and (iii) SumAA: sum of SumDEHP, MBP, MiBP and MBzP, which is an estimate of exposure with known anti-androgenic activity in experimental and/or epidemiological studies [Marie, Vendittelli and Sauvant-Rochat (2015), Marsee et al. (2006)]. We recognized that the overlap of the EDC mixture grouping would lead to dependent results for associations. However, our primary focus on the summary measures would reduce the burden of adjustment of multiple comparisons in examining each phthalate concentration individually. Hence, grouping EDCs allowed us to facilitate the interpretation of the association results.

Potential confounders. Several key adjustment factors suggested by our collaborators were considered in the association analysis of growth dynamics with the exposure to EDCs. For 213 mother-infant pairs, the considered confounding variables [Mean (SD) or \%] are as follows: cohort indicator (22.5\% pairs from cohort 2 and $77.5 \%$ pairs from cohort 3 ), infant's sex (53.1\% girls), breast-fed for 6 month $(62 \%$ yes), birth weight [3.2 (0.4) kg], gestational age [39.1 (1.1) weeks], mother's age at-birth [27.6 (5.6) years], years of education [11.1 (2.8)], marital status (88.3\% for married/with partner and $11.7 \%$ of single/separated/divorced/widow), number of previous pregnancies $(29.1,7.5,63.4 \%$ for $0,1,2+$, respectively), and current/previous smoking ( $10.8 \%$ yes).

\section{Statistical analysis.}

3.1. Bayesian modeling of dynamics. To incorporate the Newton's law of motion in the infant BMI growth modeling, we propose a statistical dynamic equation based on a semiparametric stochastic velocity model, which is referred to as 
Newton's growth model (NGM) in the rest of the paper. The details of NGMs are described as follows. Let $Y_{i}\left(t_{i j}\right)$ denote the measured BMI of the $i$ th infant at time $t_{i j}, j=1,2, \ldots, J_{i}, i=1,2, \ldots, n$. For notational simplicity for the paneled longitudinal data considered in this paper, we write $t_{i j}$ as $t$ from now on. An NGM is to model continuous-time dynamics of BMI growth, consisting of the following three equations: for the $i$ th infant, the three elements of growth motion are given by

$$
\begin{aligned}
& \text { Trajectory: } & Y_{i}(t) & =U_{i}(t)+\epsilon_{i}(t), \quad \text { where } \epsilon_{i}(t) \stackrel{\text { i.i.d. }}{\sim} N\left(0, \sigma_{\epsilon}^{2}\right), \\
& \text { Velocity: } & d U_{i}(t) & =V_{i}(t) d t, \\
& \text { Acceleration: } & d V_{i}(t) & =a\left\{V_{i}(t) ; \mathbf{X}_{i}, \boldsymbol{\theta}_{i}\right\} d t+b\left\{V_{i}(t) ; \mathbf{X}_{i}, \boldsymbol{\theta}_{i}\right\} d W_{i}(t),
\end{aligned}
$$

where the trajectory equation (3.1) involves the individual mean BMI trajectory, $U_{i}(t)$, of the $i$ th infant's BMI curve with normally distributed errors $\epsilon_{i}(t)$ with mean zero and variance $\sigma_{\epsilon}^{2}$; the velocity equation (3.2) describes the mean velocity function, $V_{i}(t)$; and the acceleration equation (3.3) specifies dynamic behaviors of $V_{i}(t)$ through a stochastic differential equation (SDE), where $a\left\{V_{i}(t) ; \mathbf{X}_{i}, \boldsymbol{\theta}_{i}\right\}$ is a drift term for a large-scale acceleration and $b\left\{V_{i}(t) ; \mathbf{X}_{i}, \boldsymbol{\theta}_{i}\right\}$ is a diffusion term for an instantaneous fluctuation of acceleration, and $W_{i}(t)$ is assumed to the standard Wiener process satisfying $d W_{i}(t) \sim N(0, d t)$. These two terms, $a(\cdot)$ and $b(\cdot)$, are assumed to be functions of observed covariates $\mathbf{X}_{i}$ and subject-specific parameters $\boldsymbol{\theta}_{i}$. In the above NGM specification, equation (3.3) may be regarded as a prior for velocity function $V_{i}(t)$. According to Wahba (1978), the resulting estimation of the $U_{i}(t)$ from the NGM (3.1)-(3.3) is closely related to the estimation of the $U_{i}(t)$ from nonparametric smoothing splines.

The NGM equation (3.3) plays an important role in the modeling of growth dynamics, which enables us to examine whether, and to what extent, covariates of environmental exposures affect infant's growth acceleration, and consequently infant's growth trajectory. Specifically, to perform the association analysis, we specify the Ornstein-Uhlenbeck (OU) process in the equation (3.3). The OU process may be expressed as, with covariates being suppressed, $d V_{i}(t)=$ $-\rho_{i}\left(V_{i}(t)-\bar{v}_{i}\right) d t+\sigma_{\xi} d W_{i}(t)$.

The OU process has several properties particularly appealing to our growth data analysis. First, as shown in Figure 3(c) and (f), the resulting BMI growth curves $U_{i}(t)$ from the OU exhibit proper shapes highly aligned with those reported by WHO for the world population of infants and toddlers [see Figure S.1 in the Supplementary Material [Baek, Zhu and Song (2019)]]. Second, in the OU process above, acceleration, $A_{i}(t)=d V_{i}(t) / d t$, and velocity are characterized by two parameters: the rate parameter $\rho_{i}(>0)$ and stable level parameter $\bar{v}_{i}$. The rate parameter $\rho_{i}$ controls acceleration which affects the speed of the velocity process $V_{i}(t)$ moving toward a stable velocity level $\bar{v}_{i}$ as shown in Figure 3(a)-(b). The larger the $\rho_{i}$, the faster the initial velocity reaches a stable level $\bar{v}_{i}$. 
(a)

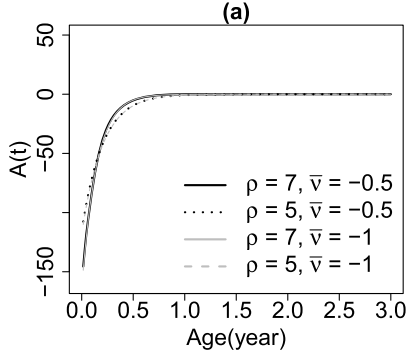

(d)

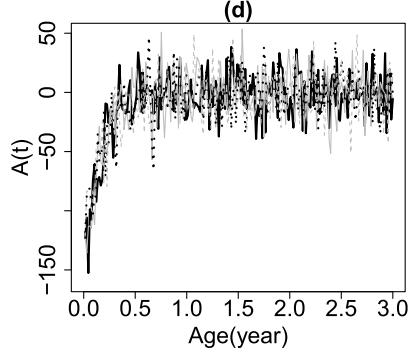

(b)

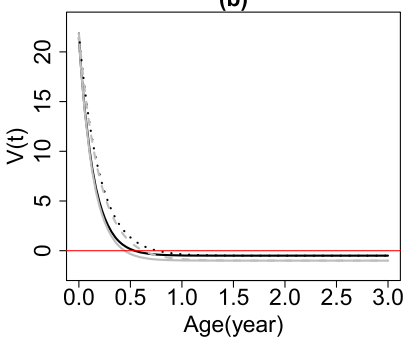

(e)

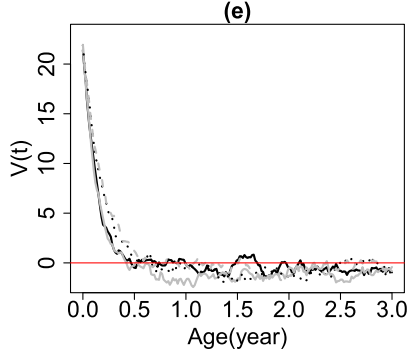

(c)

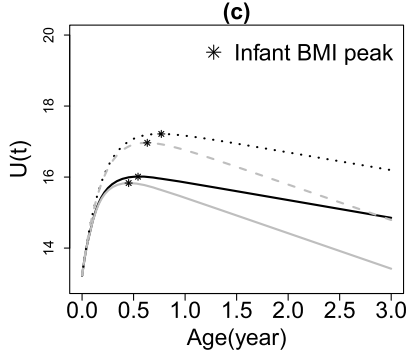

(f)

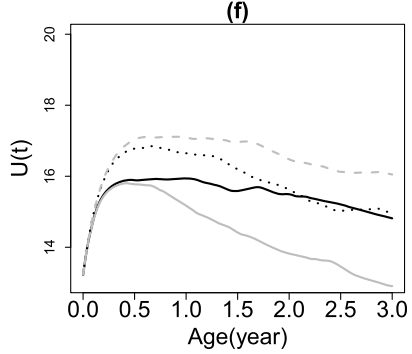

FIG. 3. Illustration of realizations from the Ornstein-Uhlenbeck $(O U)$ process. Mean growth acceleration $A(t)$, velocity $V(t)$ and trajectory $U(t)$ with $\sigma_{\xi}^{2}=0$ (a)-(c) and $\sigma_{\xi}^{2}=5$ (d)-(f). Growth mean trajectory curve with $\sigma_{\xi}^{2}=0$ (c) and $\sigma_{\xi}^{2}=5$ (f).

In Figure 3(a)-(c), we also see that with $\sigma_{\xi}^{2}=0$ and a fixed common stable velocity $\bar{v}_{i}, V_{i}(t)$ moves to its stable velocity $\bar{v}_{i}$ faster with a higher rate parameter $\rho_{i}$, which consequently results in an earlier infant peak with a lower BMI (or slimmer infant) at the age of the peak for those with $\bar{v}_{i}<0$ during the study period. In addition, the impact of the instantaneous variance $\sigma_{\xi}^{2}$ of the OU process is demonstrated by Figure 3(d), (f) indicating clear short-term variations on the growth dynamics. It is worth reiterating the point that the infant BMI peak is one of the most important growth milestones, and so a significantly delayed timing of this milestone is detrimental for health later in life.

One of our new contributions to the growth curve analysis is an important extension of the classical OU process, which allows both rate parameter $\rho_{i}$ and stable velocity $\bar{v}_{i}$ to vary across infants with environmental exposure of interest (e.g., BPA or phthalates), denoted by $X_{i}$, as well as confounding factors $\mathbf{Z}_{i}$. Specifically, due to the positivity of the rate parameter $\rho_{i}$, we propose the following association models for the two parameters in the OU process:

$$
\begin{aligned}
\log \left(\rho_{i}\right) & =\beta_{0}+\beta_{1} X_{i}+\mathbf{Z}_{i}^{T} \boldsymbol{\beta}_{z}+b_{\rho i}, \quad \text { where } b_{\rho i} \stackrel{\text { i.i.d. }}{\sim} N\left(0, \sigma_{\rho}^{2}\right), \\
\bar{v}_{i} & =\gamma_{0}+\mathbf{Z}_{i}^{T} \boldsymbol{\gamma}_{z}+b_{\bar{v} i}, \quad \text { where } b_{\bar{v} i} \stackrel{\text { i.i.d. }}{\sim} N\left(0, \sigma_{\bar{v}}^{2}\right),
\end{aligned}
$$

where normally distributed random intercepts $b_{\rho i}$ and $b_{\bar{v} i}$ capture subject-specific variabilities. Here, EDC $X_{i}$ is included in equation (3.4), not in equation (3.5), be- 
cause the rate parameter $\rho_{i}$ characterizes the initial growth force at birth, through which we are able to assess if, and how, $\rho_{i}$ is altered by in utero exposure to EDCs. Our analysis in this paper explicitly models the rate parameter $\rho_{i}$ as a function of exposure variables in order to address the $\mathrm{DOHaD}$ hypothesis. In contrast, the parameter $\bar{v}_{i}$ concerns the stable level occurring on average approximately one year after birth, and so it is not immediately adjacent to in utero conditions. Scientifically, this rate parameter determines the timing of the infant BMI peak for an individual with $\bar{v}_{i}<0$ during the study period of birth to 3 years old. It is worth pointing out that when the infant BMI peak exists for each subject in the cohort, constraining $\bar{v}_{i}$ to be negative gives rise to a model ensuring the existence of infant BMI peak during the age period considered, which may help gain statistical power and better interpretations of the results. In this study, $\bar{v}_{i}$ was not constrained to be negative because some infants may possibly not reach the BMI peak during the study period.

3.2. Incorporation of WHO data. Several world population-level infant BMI growth patterns have been reported in the literature, and among those, the worldpopulation curves given by WHO present most relevant knowledge to our analysis. Given that human growth is a highly regulated biological process, incorporating such population-level information into our analysis can help us improve our estimation of infant growth curves. Using WHO information is particularly appealing in our setting where we worked with regularly-spaced longitudinal data with relatively small number of visit times. In our proposed NGM, the initial distributions of growth dynamics at-birth played an important role in constraining the space of potential growth trajectories, over which the statistical solution is searched. Hence, we proposed to utilize the relevant existing knowledge in the specification of the initial distributions for robust and reliable parameter estimation, in a similar spirit of empirical Bayes.

The WHO population-level growth BMI data with two month sampling intervals up to two years for boys and girls are provided by WHO [WHO (2016)]. We fitted our NGM to such WHO data and obtained the estimates for parameters in the initial distributions of $\left[U\left(t_{0}\right), V\left(t_{0}\right)\right]^{T}$ for boys and girls, separately (see Figure S.1 in the Supplementary Material [Baek, Zhu and Song (2019)]). Note that the initial distribution of the trajectory and velocity $\left[U_{i}\left(t_{0}\right), V_{i}\left(t_{0}\right)\right]^{T} \sim N_{2}\left(\boldsymbol{m}_{0}, \boldsymbol{C}_{0}\right)$, with mean vector $\boldsymbol{m}_{0}=\left(m_{01}, m_{02}\right)^{T}$ and covariance matrix $\boldsymbol{C}_{0}=\left(c_{l k}\right)_{2 \times 2}, l, k=1,2$. It is worth noting that from the WHO summary statistics data only the mean vector $\boldsymbol{m}_{0}$ and the variance $c_{11}$ were estimable, namely; $\left(\hat{m}_{01}, \hat{m}_{02}\right)^{T}=(13.2,31)^{T}$ for boys and $(13.1,23)^{T}$ for girls, with $\hat{c}_{11}=0.9$ for both boys and girls. These mean estimates were adopted in the specification of the corresponding hyper-parameters of the priors in this paper. The variance of velocity $c_{22}$, however, was not estimable given the available WHO growth data which only contains the summary statistics of population BMI trajectory means and its standard deviations. Without actual 
individual data, those summary statistics were insufficient to obtain an estimate of the variance $c_{22}$.

3.3. Priors. For covariance $\boldsymbol{C}_{0}$ in the initial distribution, we specified $c_{11}=$ 10 which was about 10 times greater than the estimated variance $\hat{c}_{11}=0.9$ from the WHO data. Since there was no available data to determine plausible values of $c_{12}$ and $c_{22}$, we began with an assumption of independence between $U_{i}\left(t_{0}\right)$ and $V_{i}\left(t_{0}\right)$ at birth, namely $c_{12}=0$. Then, we calculated an empirical estimate of the velocity variance $c_{22}$ according to the infant BMI slopes between at-birth and six months of the ELEMENT data. Then, we set $c_{22}=20$, which was about twice greater than the empirical estimate. Moreover, assuming the mutual independence of $\epsilon_{i}(t)$, $b_{\rho i}, b_{\bar{v} i}$ and $W_{i}(t)$, we imposed the following noninformative priors: (i) for the variance of the error $\epsilon_{i}(t), \sigma_{\epsilon}^{2} \sim \mathrm{IG}(0.01,0.01)$, where IG stands for the inverse gamma distribution; (ii) for the parameters in equation (3.4), $\boldsymbol{\beta}=\left[\beta_{0}, \beta_{1}, \boldsymbol{\beta}_{z}\right]^{T} \sim$ $N\left(\mathbf{0}, 10^{8} \mathbf{I}\right)$ and $\sigma_{\rho}^{2} \sim \operatorname{IG}(0.01,0.01)$; (iii) the parameters in equation $(3.5), \boldsymbol{\gamma}=$ $\left[\gamma_{0}, \boldsymbol{\gamma}_{z}\right]^{T} \sim N\left(\mathbf{0}, 10^{8} \mathbf{I}\right)$ and $\sigma_{\bar{\nu}}^{2} \sim \operatorname{IG}(0.01,0.01)$; and (iv) for the instantaneous volatility parameter $\sigma_{\xi}^{2} \sim \mathrm{IG}(0.1,0.1)$.

3.4. MCMC algorithm. The MCMC method previously implemented for BSSVM was based on a data augmentation approach under the approximated transition density. Although the data augmentation approach seemed easy to use because of the conjugate forms of posterior distributions, it artificially increases number of parameters on unobserved time points. Due to the increased number of parameters, convergence rate of posterior samples could be slow, and mixing for posterior sampling would be of low quality.

In this paper, we implemented an highly efficient MCMC method that directly carried out posterior sampling by evaluating the exact transition density function, where evaluating the density function can be computationally parallelized. Because the proposed NGM can be expressed in terms of a state space model representation, justifying the model identifiability as well as the validity for parameter estimation and inference may be routinely done by applying the existing theory of the state space models [Zhu, Taylor and Song (2011)]. Our posterior sampling approach was based on a shrinkage slice sampling [Agarwal and Gelfand (2005)], which was extended by Neal (2003). See the MCMC details in Section S.4 and S.5 in the Supplementary Material [Baek, Zhu and Song (2019)]. Our proposed MCMC method is generally applicable for any B-SSVM which is a reduced model of the NGM. Our proposed implementation of the MCMC algorithm has shown a substantial improvement in the computational efficiency in drawing posterior samples, in comparison to the standard B-SSVM implementation. For instance, our method enabled us to draw posterior samples of certain parameters 1000 times faster over the standard B-SSVM implementation, and this improvement made the proposed method practically useful. See more details in Section S.6 in the Supplementary Material [Baek, Zhu and Song (2019)]. 


\section{ELEMENT data analysis.}

4.1. Preliminary analysis. Before we began with our analysis of the ELEMENT data, we performed an exploratory data analysis for the association between infant's BMI growth rate and individual log-transformed EDC or mixture of EDCs at each trimester. We first calculated empirical BMI growth velocities at 6 months, $\tilde{V}_{i}$, for each infant using data at birth, 3 and 6 months. Then, we fitted a simple linear regression model, $\tilde{V}_{i}=\eta_{0}+\eta_{1} X_{i}+\epsilon_{i}$, to examine the association of the 6-month BMI growth velocity with EDC exposure $X_{i}$. Figure 4(a) shows the estimates, $\hat{\eta}_{1}$, where the $x$-axis accommodates 10 single EDCs and 3 mixtures of EDCs over the three trimesters. We noticed that only during the first trimester, the 6-month BMI growth velocity was positively associated with in utero exposure to EDCs. This positive association suggested that during the first 6 months the mean BMI tended to increase faster with more severe exposure to EDCs. However, no significant signals were detected in the second and third trimesters.

4.2. Model-based analysis. With those specified priors aforementioned in Section 3.3, we fitted the proposed NGM with the confounding variables discussed in Section 2, which were sequentially added into models in the equations (3.4)(3.5). We ran the MCMC algorithm via the shrinkage slice-sampling over 5000 iterations, with the first 1000 iterations being discarded for the burn-in, and the remaining 4000 draws being retained for the subsequent Bayesian analysis. It took approximately 7 minutes in run-time for each candidate model on a PC with Intel (R) Xeon (R) CPU E5-1620 v3 (3.5 Hz). We used the classical deviance information criterion (DIC) to assess whether there is variability of both individual rate and stable velocity and to select confounding factors that do not vary by trimesters. Our model fitness assessment indicated that rate and stable velocity vary by individuals and provided the following confounding factors that do not vary by trimesters:

(a)

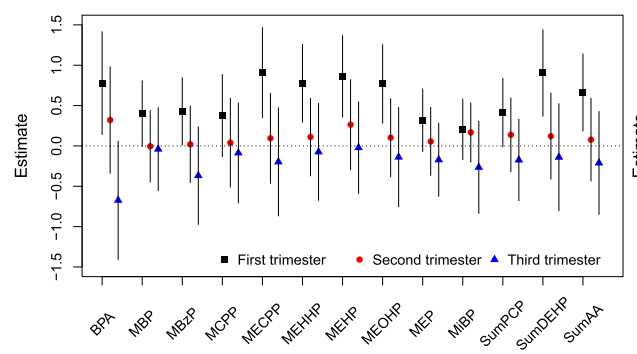

(b)

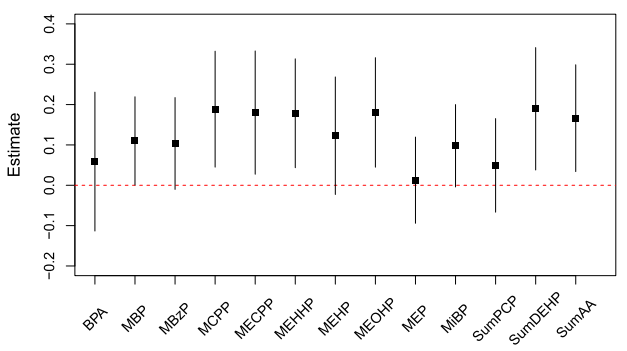

FIG. 4. (a) Preliminary results of associations $\left(\hat{\eta}_{1}\right)$ between the 6-month BMI growth velocity and in utero exposure to a single EDC or a mixture of EDCs over the three trimesters. (b) Estimated associations $\left(\hat{\delta}_{1}\right)$ from the SITAR model with a single EDC or a mixture of EDCs for the first trimester (see Section 4.3). Enlarged figures are available in the Supplementary Material [Baek, Zhu and Song (2019)]. 
(a)

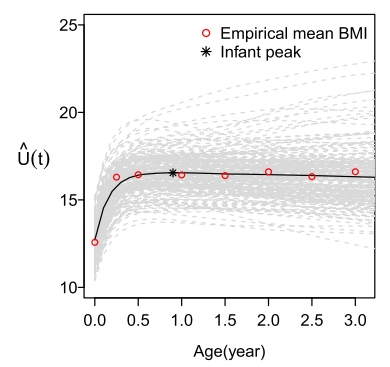

(b)

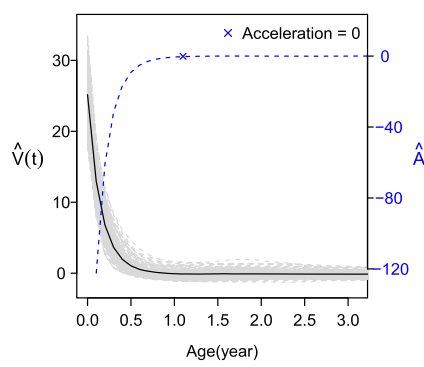

(c)

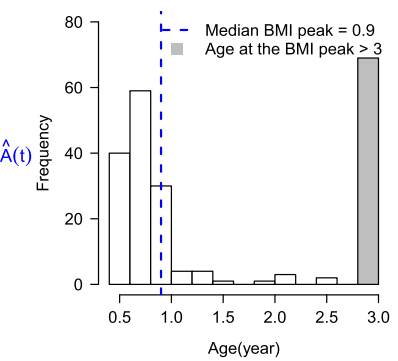

FIG. 5. (a) Estimated overall mean BMI trajectory (bold line) and individual mean BMI trajectories (grey dash lines). (b) Estimated overall mean BMI velocity (bold line) and acceleration (bold dash line) with individual mean BMI velocities (grey dash lines). (c) Histogram for timing of the infant BMI peak across infants with those who did not achieve the milestone at age 3 or younger (shaded bar).

cohort, infant's sex, breast feeding up to 6 months, birth weight, gestational age and mother's age at birth. We assessed validity of the proposed model and checked convergence of drawn posterior samples (see Section S.7 in the Supplementary Material [Baek, Zhu and Song (2019)]).

Figure 5 shows the results of estimated growth dynamics from the selected model. As shown in Figure 5(a), the estimated mean BMI trajectory appeared to fit well with the BMI growth trajectories; it rose rapidly during the first 6 months after birth, and then achieved the BMI infant peak at an average of 10.8 months with $95 \%$ credible interval $(\mathrm{CI}),(9.6,14.4)$. Note that the timing of the infant BMI peak corresponds to the time $t$ satisfying $E\left[\hat{V}_{i}(t)\right]=0$ for those with $\bar{v}_{i}<0$ over the period of $0-3$ years old; see Figure 5(b). It was clear that infants with higher or lower BMI at birth tended to stay at the respective BMI rank over time, which indicated strong within-subject correlations and noticeable variabilities across infants. The mean BMI velocity curve began to decay around the 11th months and then became gradually stabilized due largely to rapid muscle development soon after infants started to walk and become more physically active. Figure 5(c) indicated that the distribution of individual timing of the infant BMI peak was highly right skewed, suggesting variable growth tempos during this most important growth period of early human life. The majority of infants achieved their infant peak between 6 and 12 months, while there were a noticeable number of infants who experienced delayed infant peak in comparison to the average age of infant peak at 11 months. An interesting finding was that 69 infants [the shaded bar in Figure 5(c)] among 213 did not achieve their infant peak before 3 years (i.e., $\bar{v}_{i}>0$ ).

Figure 6 shows four representative examples of estimated infant BMI trajectories and the estimated corresponding ages of achieving infant BMI peak, where a comparison was made to the estimated curves given by various models (see Section 4.3). The infant in Figure 6(a) achieved the BMI peak earlier than the average age of 10.8 months, while the infant in Figure 6(d) experienced a clear delay 
(a)

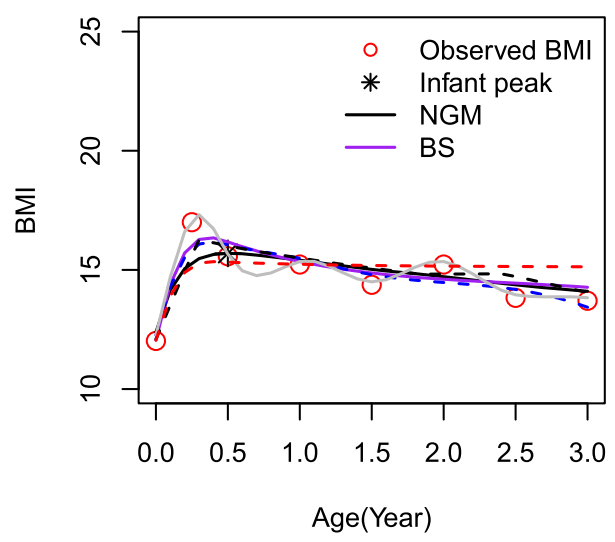

(c)

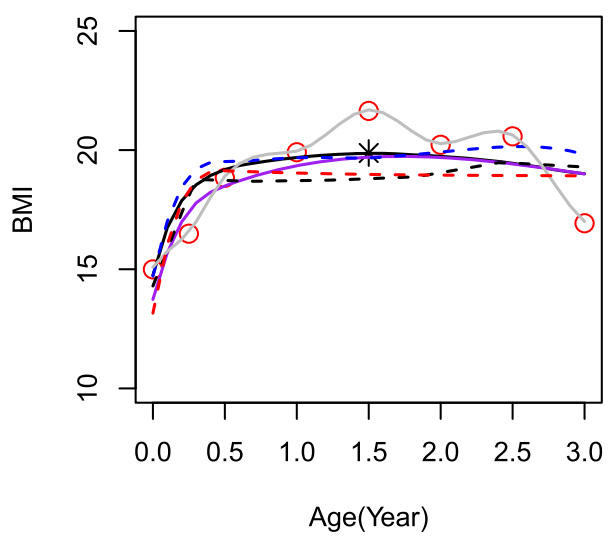

(b)

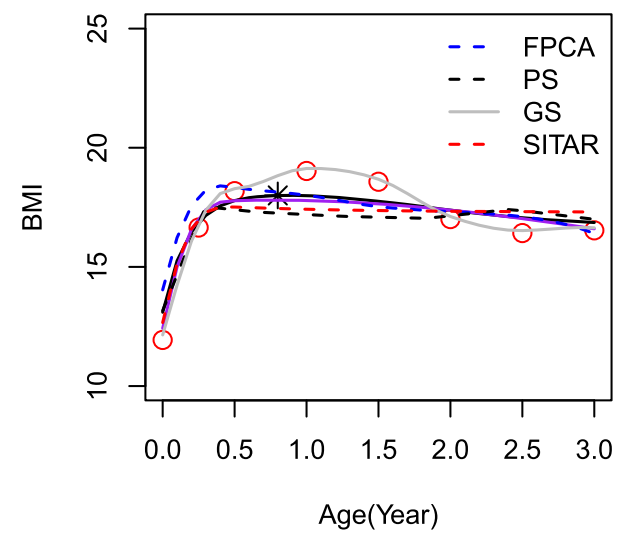

(d)

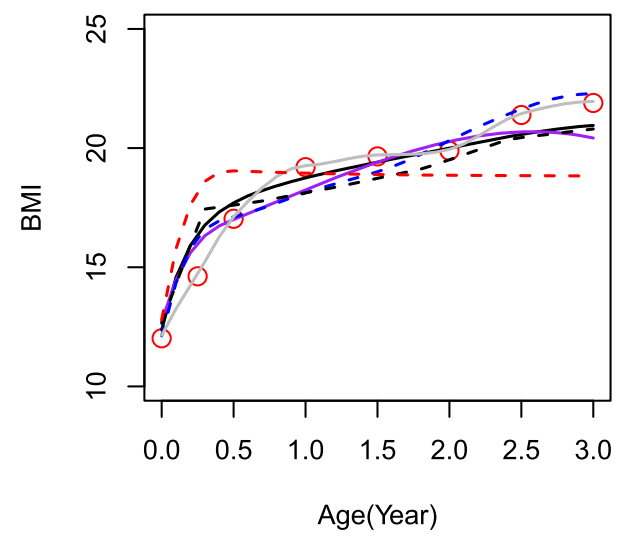

FIG. 6. Estimated individual mean BMI trajectories across various models: NGM, BS, FPCA, PS, GS, SITAR. Infant BMI peak was achieved at age around (a) 6 months, (b) 10 months, (c) 18 months, while (d) infant BMI steadily rose with no peak (or delayed BMI peak after 3 years).

to achieve the milestone before 3 years old. In regard to the $\mathrm{DOHaD}$ hypothesis in environmental health sciences, it is of great importance to understand whether such large discrepancy of tempo for the infant peak was associated with prenatal environmental exposure to EDC. Our method in this paper enabled us to answer such an important question. We stratified the infants by the estimated median (10.8 months) infant peak age and then compared their mean of the first trimester exposure to EDCs between the two groups. Note that the group with their age peak $>10.8$ months also includes infants with their estimated $\bar{v}_{i}>0$. As shown in Table 1, the mean exposure to EDCs for those with delayed infant peak tended higher than for those with early infant peak. Based on the stratification by the median of 
TABLE 1

Summary statistics for the first trimester exposure to 10 single EDCs and 3 mixtures of EDCs between two groups of infants stratified by the median (10.8 months) age at the infant BMI peak. $P$-value was calculated based on two sample t-test

\begin{tabular}{|c|c|c|c|c|c|}
\hline \multirow{2}{*}{$\begin{array}{l}\text { The first trimester } \\
\text { exposure }\end{array}$} & \multicolumn{2}{|c|}{ Age peak $\leq 10.8$ months } & \multicolumn{2}{|c|}{ Age peak $>10.8$ months } & \multirow[b]{2}{*}{$P$-value } \\
\hline & Mean & SD & Mean & SD & \\
\hline \multicolumn{6}{|l|}{ Single EDC } \\
\hline BPA & -0.367 & 0.185 & 0.833 & 0.437 & $<0.001$ \\
\hline MBP & -0.049 & 0.906 & 0.088 & 0.918 & 0.309 \\
\hline MBzP & 3.944 & 1.413 & 4.165 & 1.403 & 0.287 \\
\hline МCPP & 0.788 & 1.360 & 1.055 & 1.377 & 0.186 \\
\hline MECPP & -0.056 & 1.071 & 0.254 & 1.039 & 0.047 \\
\hline MEHHP & 3.269 & 0.967 & 3.487 & 1.063 & 0.145 \\
\hline MEHP & 2.661 & 1.156 & 2.923 & 1.126 & 0.120 \\
\hline MEOHP & 1.554 & 1.159 & 1.573 & 0.964 & 0.901 \\
\hline MEP & 2.034 & 1.147 & 2.302 & 1.123 & 0.110 \\
\hline MiBP & 5.035 & 1.412 & 4.824 & 1.496 & 0.325 \\
\hline \multicolumn{6}{|l|}{ Mixture of EDCs } \\
\hline SumPCP & -0.287 & 1.531 & 0.154 & 1.499 & 0.049 \\
\hline SumDEHP & 5.523 & 1.288 & 5.409 & 1.385 & 0.562 \\
\hline SumAA & 4.022 & 1.017 & 4.222 & 1.020 & 0.182 \\
\hline
\end{tabular}

ages at the peak, the first trimester exposure to BPA, MECPP and SumPCP was associated with the delayed infant peak. In additional comparisons for those who had a peak $\left(\bar{v}_{i}<0\right)$ versus those without a peak $\left(\bar{v}_{i}>0\right)$, BPA remained significant.

The NGM with the regression models in equations (3.4)-(3.5) allowed to account for the confounding relevant to the growth acceleration, comparing between trajectory/velocity curves between infants. Table 2 shows that the estimated effects of all single EDCs in the first trimester were negative, suggesting that in utero exposure to a single toxicant was inversely associated with the growth acceleration of infant BMI; the inverse association with the acceleration indicates delayed infant BMI peak and higher BMI at the peak (see Figure S.2 in the Supplementary Material [Baek, Zhu and Song (2019)]). We estimated the posterior probability of the inverse association, $P\left(\beta_{1}<0 \mid \mathbf{Y}, \mathbf{X}, \mathbf{Z}\right)$ according to $\sum_{k=1}^{4000} I\left(\tilde{\beta}_{1}^{(k)}<0\right) / 4000$, where $\tilde{\beta}_{1}^{(k)}$ is a drawn posterior sample of $\beta_{1}$ at the $k$ th iteration. The estimated posterior probabilities $P\left(\beta_{1}<0 \mid \mathbf{Y}, \mathbf{X}, \mathbf{Z}\right)$ were $0.962,0.967,0.969,0.968$ for the first trimester exposure to MBP, MEHHP, MEOHP, MiBP, respectively, and 0.994 for the second trimester exposure to MiBP.

In regard to mixtures of EDCs, the estimated effects in the first trimester were negative as well. We found that the first trimester exposure to SumAA was associated with the BMI growth acceleration, with the estimated posterior proba- 
TABLE 2

Estimated regression coefficients $\beta_{1}$ with $95 \%$ credible intervals concerning in utero exposures to a single EDC and mixtures of EDCs in the selected NGM. Here, $p^{*}$ denotes the estimated posterior probability $P\left(\beta_{1}<0 \mid \mathbf{X}, \mathbf{Y}, \mathbf{Z}\right)$

\begin{tabular}{|c|c|c|c|c|c|c|c|c|c|c|c|c|}
\hline \multirow[b]{2}{*}{ Parameter } & \multicolumn{4}{|c|}{ First trimester $(n=185)$} & \multicolumn{4}{|c|}{ Second trimester $(n=184)$} & \multicolumn{4}{|c|}{ Third trimester $(n=180)$} \\
\hline & $\mathbf{5 0 \%}$ & $2.5 \%$ & $97.5 \%$ & $p^{*}$ & $50 \%$ & $2.5 \%$ & $97.5 \%$ & $p^{*}$ & $50 \%$ & $2.5 \%$ & $97.5 \%$ & $p^{*}$ \\
\hline \multicolumn{13}{|l|}{ Single EDC } \\
\hline $\mathrm{BPA}$ & -0.044 & -0.112 & 0.020 & 0.913 & -0.006 & -0.073 & 0.063 & 0.570 & 0.044 & -0.028 & 0.118 & 0.125 \\
\hline MBP & -0.040 & -0.084 & 0.006 & 0.962 & -0.005 & -0.049 & 0.037 & 0.587 & 0.022 & -0.026 & 0.068 & 0.184 \\
\hline MBzP & -0.017 & -0.062 & 0.025 & 0.787 & -0.006 & -0.052 & 0.041 & 0.602 & 0.045 & -0.015 & 0.105 & 0.070 \\
\hline MCPP & -0.038 & -0.090 & 0.018 & 0.914 & 0.002 & -0.054 & 0.059 & 0.474 & 0.022 & -0.037 & 0.081 & 0.220 \\
\hline MECPP & -0.055 & -0.121 & 0.003 & 0.966 & -0.034 & -0.091 & 0.023 & 0.880 & 0.016 & -0.044 & 0.076 & 0.296 \\
\hline MEHHP & -0.052 & -0.106 & 0.003 & 0.967 & -0.018 & -0.065 & 0.028 & 0.761 & 0.012 & -0.047 & 0.067 & 0.334 \\
\hline MEHP & -0.038 & -0.097 & 0.017 & 0.924 & -0.018 & -0.072 & 0.040 & 0.734 & -0.002 & -0.059 & 0.051 & 0.526 \\
\hline MEOHP & -0.051 & -0.106 & 0.003 & 0.969 & -0.017 & -0.067 & 0.034 & 0.753 & 0.021 & -0.034 & 0.076 & 0.223 \\
\hline MEP & -0.003 & -0.047 & 0.035 & 0.556 & -0.017 & -0.059 & 0.026 & 0.782 & 0.022 & -0.019 & 0.063 & 0.144 \\
\hline MiBP & -0.036 & -0.073 & 0.002 & 0.968 & -0.045 & -0.080 & -0.010 & 0.994 & -0.008 & -0.067 & 0.048 & 0.603 \\
\hline \multicolumn{13}{|c|}{ Mixture of EDCs } \\
\hline SumPCP & -0.017 & -0.063 & 0.030 & 0.772 & -0.016 & -0.063 & 0.034 & 0.743 & 0.028 & -0.019 & 0.073 & 0.120 \\
\hline SumDEHP & -0.056 & -0.117 & 0.002 & 0.971 & -0.027 & -0.084 & 0.029 & 0.833 & 0.013 & -0.047 & 0.070 & 0.331 \\
\hline SumAA & -0.055 & -0.108 & -0.004 & 0.985 & -0.021 & -0.074 & 0.033 & 0.791 & 0.019 & -0.042 & 0.081 & 0.271 \\
\hline
\end{tabular}




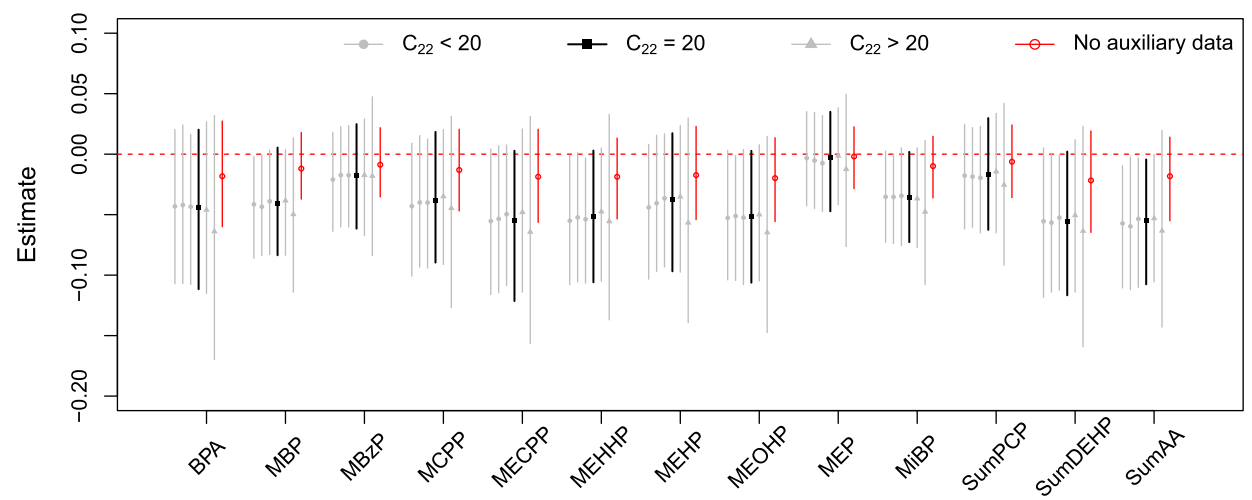

FIG. 7. Estimates of $\beta_{1}$ in equation (3.4) with $95 \%$ credible interval for the first trimester exposure to EDCs. The estimates were obtained and displayed according to the sequence of the variances of initial velocity $c_{22} \in\left\{5,10,15,20,40,10^{3}\right\}$ and no WHO auxiliary data with setting $m_{01}=0, c_{11}=10^{8}, m_{02}=0, c_{22}=10^{8}$. Note that the data analysis was performed with $c_{22}=20$.

bility $P\left(\beta_{1}<0 \mid \mathbf{Y}, \mathbf{X}, \mathbf{Z}\right)=0.985$. Also, it is noted that the estimated posterior probability of the inverse association was 0.971 for the first trimester exposure to SumDEHP. The results are all in agreement with those reported in the preliminary association analysis in Section 4.1. The effect of SumPCP, which is sum of MBP and MEP, was probably downsized by MEP whose effect was found to be close to the null with the posterior probability of the inverse association equal to 0.556 .

We performed a sensitivity analysis with varying variance of the initial velocity $c_{22}$, with the focus on the robustness pertaining to estimation and inference for $\beta_{1}$, the key parameter of association with the first trimester exposure to EDCs. We assigned value $c_{22} \in\left\{5,10,15,40,10^{3}\right\}$. Furthermore, we investigated consequences of fitting models without any WHO auxiliary data. Note that the data analysis above used $c_{22}=20$ which has been marked by solid black lines in Figure 7. As shown in Figure 7, the point estimates and their $95 \% \mathrm{CI}$ of the association parameter $\beta_{1}$ appeared to be rather consistent unless $c_{22}$ is too large $\left(10^{3}\right)$. Note that when strong prior variance $c_{22} \in\{5,10\}$ were used, the resulting $95 \%$ CIs of $\beta_{1}$ turned out to be similar and comparable. We reported more thorough simulation experiments to examine the sensitivity of the prior specification on the analysis results in Section 5. Without WHO auxiliary data, all parameter estimates of EDCs went toward the null as similarly observed in Section 5. Overall, we learned from a simulation study in Section 5 that the proposed Bayes inference tended to be valid to the choice of the prior values of $c_{22}$.

4.3. Comparison to existing models. In the current literature, there has been a limited arsenal developed to analyze the association between environmental exposure and growth dynamics. To run a confirmatory analysis we considered the 
following nonparametric or growth models: BS-model: a B-spline linear mixed effects model [Rice and Wu (2001)]; FPCA-model: a functional principal components analysis for sparse data [Yao, Müller and Wang (2005)]; PS-model: a penalized linear spline mixed effects model [Durbán et al. (2005)]; GS-model: nonparametric gradients for sparsely observed functional data [López-Pintado and McKeague (2013)]; SITAR-model: the SuperImposition by Translation And Rotation proposed by Cole, Donaldson and Ben-shlomo (2010). A shared feature among the BS-, FPCA- and PS-model pertains to the use of pre-specified basis functions to model the mean trajectories of growth, in which the BS-, FPCAand PS-model uses, respectively, B-spline basis functions, Karhunen-Loève basis expansion and penalized linear splines for the underlying subject-specific trajectories. The GS-model directly estimates individual-level empirical velocities from which we estimated trajectories by simply numerically integrating these empirical velocities. The SITAR-model takes the form: $E\left[Y_{i}(t)\right]=a_{i}+h((t-$ $\left.b_{i}\right) / \exp \left(-c_{i}\right)$ ), where $Y_{i}(t)$ is the BMI for infant $i$ at time $t, h(t)$ is a nonparametric smoothing function approximated by natural cubic splines and $a_{i}, b_{i}$ and $c_{i}$ are infant-specific "shift" and "scale" random effects corresponding to size, timing of peak velocity and velocity, respectively. Note that the BS-, FPCA-, PS- and SITAR-model directly specify the mean BMI growth curve and the GS-model focuses the mean BMI velocity curve. Only our proposed NGM explicitly addresses three key growth dynamics including trajectory, velocity and acceleration in one growth dynamic system.

In this comparison analysis, we fitted these models to subjects observed at all 8 visits $(n=116)$ because the GS-model only allowed to use subjects equally observed over time (i.e., a rectangular type of data). Then, we performed a crossvalidation $(\mathrm{CV})$ analysis to evaluate the performance of prediction among these models. We removed each time point, $t_{j}, j=2, \ldots, 7$, of all subjects and predicted its mean at that time point by a fitted model. The CV errors were calculated by $C V\left(t_{j}\right)=\sum_{i=1}^{n}\left(Y_{i, t_{j}}-\hat{Y}_{i,-t_{j}}\right)^{2} / n$, which was not evaluated at $j=1$ (at-birth) or $j=8$ (36 months) to avoid extrapolation. Since the performance of each model may depend on the selection of knots, we searched the best model of each case via appropriate tuning procedures (see Section S.8 in the Supplementary Material [Baek, Zhu and Song (2019)]).

As shown in Table 3, the BS-model provided the least overall CV errors and was followed by our NGM. Looking more closely, we found that for each time point, the BS-model was the best at $j=2,3$ (3 and 6 months); NGM was the best at $j=4$ (12 months); the FPCA-model was the best at $j=5,6,7(18,24,30$ months). Figure 6 shows that individual fitted trajectories estimated across various methods obtained from these models. The GS-model provided the wiggliest curve (i.e., no advantage of de-noising). The SITAR-model tends to fail capturing individual specific features. Individual trajectories from NGM seems slightly smoother than from the BS-, FPCA- and PS-model while NGM still enables to capture individual curves. However, the most important feature of NGM is that researchers 
TABLE 3

Cross-validation errors across methods at each time point $t_{j}, j=2, \ldots, 7$. $C V\left(t_{j}\right)=\sum_{i=1}^{n}\left(Y_{i, t_{j}}-\hat{Y}_{i,-t_{j}}\right)^{2} / n$. The $C V$ result is listed by ascending order of the overall $C V$

\begin{tabular}{lccccccc}
\hline & \multicolumn{7}{c}{$\boldsymbol{C} \boldsymbol{V}\left(\boldsymbol{t}_{\boldsymbol{j}}\right)$} \\
\cline { 2 - 7 } Model & $\boldsymbol{j}=\mathbf{2}$ & $\boldsymbol{j}=\mathbf{3}$ & $\boldsymbol{j}=\mathbf{4}$ & $\boldsymbol{j}=\mathbf{5}$ & $\boldsymbol{j = 6}$ & $\boldsymbol{j = 7}$ & Overall \\
\hline BS & 1.47 & 1.14 & 1.49 & 1.56 & 1.03 & 0.75 & 1.24 \\
NGM & 1.81 & 1.24 & 1.40 & 1.63 & 1.05 & 0.82 & 1.33 \\
SITAR & 2.12 & 1.44 & 1.42 & 1.58 & 1.22 & 1.22 & 1.50 \\
FPCA & 2.39 & 2.01 & 1.44 & 1.59 & 1.00 & 0.66 & 1.51 \\
PS & 4.64 & 1.42 & 1.42 & 1.52 & 1.07 & 0.84 & 1.82 \\
GS & 3.23 & 3.80 & 2.70 & 2.13 & 1.43 & 0.90 & 2.36 \\
\hline
\end{tabular}

can examine how growth acceleration is associated with potential risk factors and capture the structural change-point corresponding to the growth milestone. For instance, NGM is able to directly indicate that subjects reached the infant BMI peak through the stable velocity (i.e., $\bar{v}_{i}>0$ ). In addition, our NGM can be used to make prediction of growth beyond the range of observations.

Furthermore, in the SITAR-model, we argue that the role of the timing parameter $b_{i}$ is somewhat similar to the rate parameter $\rho_{i}$ in equation (3.4). Thus, we considered an association analysis model fitted in the first trimester data $(n=185)$ : $E\left[b_{i}\right]=\delta_{0}+\delta_{1} X_{i}+\mathbf{Z}_{i}^{T} \boldsymbol{\delta}_{z}$, where a positive association $\delta_{1}$ indicates delayed timing of peak velocity, and vector $\mathbf{Z}_{i}$ consisted of the confounders used in our selected model.

As shown in Figure 4(b), the SITAR-model unveiled that the peak timing parameter $b_{i}$ was positively associated with all single EDCs as well as mixtures of EDCs. These findings fully agreed with those found by our NGM. That is, in utero exposure to EDCs in the first trimester was associated with the delayed infant BMI peak. Although the two parameters, the timing parameter $b_{i}$ in the SITAR-model and the rate parameter $\rho_{i}$ in our dynamic growth model, do not measure exactly the same growth dynamics, we found conformal directions of association of the infant peak timing with the first trimester exposure to EDCs. Our experience with the use of SITAR-models suggested that the SITAR-model implicitly assumed that individual infants must have achieved their infant peaks during a study time period (e.g., 0-3 years) because this model required a shape of the mean trajectory to follow that of a quadratic function [see Figure 6(a)-(d)]. This functional assumption of the SITAR-model might sometimes cause convergence failure. It is more critical to notice that from Figure 6(a)-(d), the SITAR-model seemed to lack the flexibility of capturing individual infant's growth trajectories, as opposed to the fact that our proposed NGM showed great adaptability to infant's growth patterns. 
5. Simulation experiment. To better understand the proposed Bayesian approach, we performed simulation studies with the following two major objectives: (1) how auxiliary knowledge about the growth condition (e.g., WHO data) may improve estimation of the association parameters of interest; (2) how, and to what extent, the smoothness level $\sigma_{\xi}^{2}$ imposed for the growth curve affects inference for the parameters in the acceleration equation (3.4).

To simulate data that mimic the ELEMENT longitudinal data, we first fitted our proposed NGM without any EDC $X_{i}$ and confounders $\mathbf{Z}_{i}$ in the two models (3.4) and (3.5). Then, we set the true values of the parameters similar to the estimates: that is, $\sigma_{\epsilon}^{2}=0.9, \sigma_{\xi}^{2}=9, \beta_{0}=1.95, \gamma_{0}=-0.25, \sigma_{\rho}^{2}=0.02, \sigma_{\bar{v}}^{2}=$ $0.1,\left[U_{i}\left(t_{0}\right), V_{i}\left(t_{0}\right)\right]^{T} \sim N\left(\boldsymbol{m}_{0}, \mathbf{C}_{0}\right)$ with $\boldsymbol{m}_{0}=\left(m_{01}, m_{02}\right)^{T}=(13,31)^{T}$ and $\mathbf{C}_{0}=$ $\operatorname{diag}\left\{c_{11}, c_{22}\right\}=\operatorname{diag}\{1,10\}$. Note that estimates of $\boldsymbol{m}_{0}$ and $\mathbf{C}_{0}$ were obtained from the boy's data. We set the true effect size for $\beta_{1}$ at -0.05 , which is in a similar magnitude to the results of MECPP, MEHHP, MEOHP, SumDEHP or SumAA reported in Table 2. Finally, we generated a baseline exposure covariate $X_{i} \sim N(0,1)$ and then a longitudinal data set of 100 subjects with 8 fixed visit times.

The MCMC was run based on the following prior specification. To examine how prior knowledge of the initial growth condition affects inference for the association parameter of interest (i.e., $\left.\beta_{1}\right)$, we set various prior means and variances for the initial distribution $\left[U_{i}\left(t_{0}\right), V_{i}\left(t_{0}\right)\right]^{T} \sim N\left(\boldsymbol{m}_{0}, \mathbf{C}_{0}\right)$ with $\boldsymbol{m}_{0}=\left(m_{01}, m_{02}\right)^{T}$ and $\mathbf{C}_{0}=\operatorname{diag}\left\{c_{11}, c_{22}\right\}$, where $m_{01} \in\{12,13,14\}, m_{02} \in\{26,31,36\}, c_{11} \in$ $\left\{1,5,10,15,20,10^{3}\right\}, c_{22} \in\left\{5,10,15,20,40,10^{3}\right\}$; plus no auxiliary data setting with $m_{01}=0, c_{11}=10^{8}, m_{02}=0, c_{22}=10^{8}$. Priors for smoothness level $\sigma_{\xi}^{2}$ were set in two scenarios: (i) $\sigma_{\xi}^{2} \sim \operatorname{IG}(0.1,0.1)$ corresponding to a low-level constraint so that the resulting space of trajectories to be searched was large, and (ii) $\sigma_{\xi}^{2} \sim \operatorname{IG}(1,0.1)$ leading to a high-level constraint, so the solution would be searched among much smoother curves than those in the first scenario. All other priors for the remaining parameters were set in the same way as in Section 3.3. We ran 1,000 simulations for each case to draw summary statistics. Within each simulation round, we carried out 1,500 MCMC iterations with the first 500 iterations as the burn-in and the remaining 1,000 iterations for statistical analysis.

Figure 8 shows the simulation results that concern the influence of the initial distribution priors on the estimation of the parameter $\beta_{1}$ that measures an association between growth acceleration and exposure under the low-level smoothness prior $\sigma_{\xi}^{2} \sim \operatorname{IG}(0.1,0.1)$. Figure $8(\mathrm{a})$ indicates that the amount of bias in estimate $\hat{\beta}_{1}$ increased when $m_{01}$ was set either lower or larger than the true value (i.e., 13) with small variance $c_{11} \in\{1,5\}$ or when the initial variance $c_{22}$ was set at a large value (i.e., $10^{3}$ ) for a vague prior. It is interesting to note that the prior mean initial velocity $m_{02}$ had nearly no effect on both estimation and inference for $\beta_{1}$ whereas it had a noticeable influence on the estimation of parameter $\beta_{0}$ [see Supplementary Figure S.4 in the Supplementary Material [Baek, Zhu and Song (2019)]]. Figure 8(b) suggests that inference for $\beta_{1}$ seemed to be influenced by the prior value $c_{22}$. With 
(a)

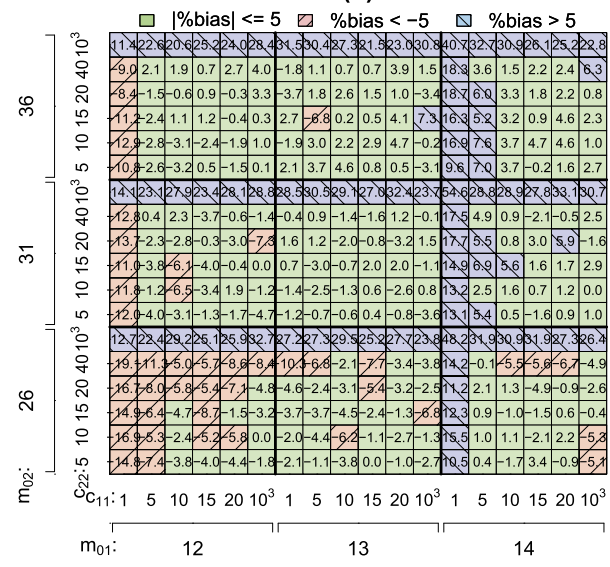

(b)

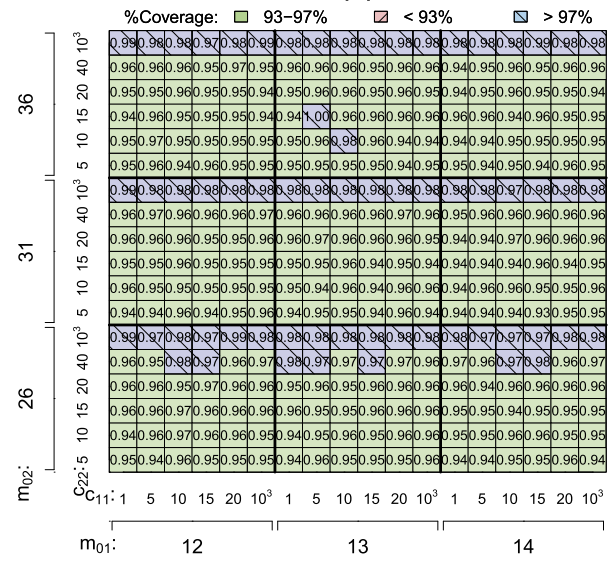

FIG. 8. Simulation results with the space of trajectories unconstrained, that is, $\sigma_{\xi}^{2} \sim \operatorname{IG}(0.1,0.1)$. (a) Bias in $\hat{\beta}_{1}$ from various priors for the initial distribution $\left[U_{i}\left(t_{0}\right), V_{i}\left(t_{0}\right)\right]^{T} \sim N\left(\boldsymbol{m}_{0}, \mathbf{C}_{0}\right)$ with $\boldsymbol{m}_{0}=\left(m_{01}, m_{02}\right)^{T}$ and $\mathbf{C}_{0}=\operatorname{diag}\left\{c_{11}, c_{22}\right\}$, where $m_{01}=\{12,13,14\}, m_{02} \in\{26,31,36\}$, $c_{11} \in\left\{1,5,10,15,20,10^{3}\right\}, c_{22} \in\left\{5,10,15,20,40,10^{3}\right\}$. (b) Corresponding coverage rates for $\beta_{1}$.

a vague prior value, $c_{22}=10^{3}$, the percent of bias in $\hat{\beta}_{1}$ increased and inference for $\beta_{1}$ tended to be conservative, namely, the credible coverage rate was greater than the $95 \%$ nominal rate. This conservatism leads to a low rate of false positives in the data analysis with a consequence of missing some true signals.

With the mean initial velocity prior $m_{02}=31$ (the true population mean velocity), if the prior variance $c_{22}$ was set to $c_{22}=10^{3}$ to result in an uninformative prior, the estimates of $\hat{\beta}_{0}, \hat{\gamma}_{0}$ may be noticeably biased, and consequently their corresponding credible coverage rates tend to be largely deviated from $95 \%$ nominal level (see Figure S.4 in the Supplementary Material [Baek, Zhu and Song (2019)]). Therefore, for such paneled longitudinal with a relatively small number of visit times, it is important to utilize scientifically relevant auxiliary data to specify informative priors for the initial distribution of $\left[U_{i}\left(t_{0}\right), V_{i}\left(t_{0}\right)\right]^{T}$, so that the proposed NGM gives adequate model parameter estimation and inference. Our strategy of prior specification in this paper has been based on the utility of the WHO population growth data. With the rationale that human growth is highly regulated, the WHO data can be applicable to the Mexican population.

Similar effects were found with the prior for the high-level smoothness $\sigma_{\xi}^{2} \sim$ IG $(1,0.1)$ that constrained the search space of trajectories, with the details available in Figure S.5 in the Supplementary Material [Baek, Zhu and Song (2019)]. The key point learned in this scenario was that this prior seemed to influence the precision of estimating variability of individual stable velocity $\sigma_{\bar{\nu}_{i}}^{2}$.

Moreover, without any auxiliary data on the initial distribution $\left(m_{01}=0\right.$, $\left.c_{11}=10^{8}, m_{02}=0, c_{22}=10^{8}\right)$, estimated $\beta_{1}$ was largely biased $(67 \%)$, with $92 \%$ coverage rate, toward the null or might not be well identified. 
We repeated this simulation study with setting true parameter $\sigma_{\xi}^{2}=0.1$ to generate much smoother individual trajectories while keeping other conditions the same. The simulation results were very similar and the prior for the high-level smoothness $\sigma_{\xi}^{2} \sim \mathrm{IG}(1,0.1)$ improved capturing the variability of individual stable velocity $\sigma_{\bar{v}}^{2}$ through an effective separation from uncertainty of short-term fluctuation.

In summary, given the regularly-spaced longitudinal data with relatively small number of visits, our proposed method seemed to have limited power in estimating variance components for the short term dynamics $\sigma_{\xi}^{2}$, the variance of random effects $\sigma_{\rho}^{2}$ and the variance of stable velocity $\sigma_{\bar{v}}^{2}$. However, estimation and inference for the key association parameter $\beta_{1}$ of scientific interest have been shown to be reliable because (i) we can utilize the WHO population data to specify the reasonable priors for the initial distribution of growth dynamics, and (ii) the sensitivity analysis with varying prior values for the variance of velocity $c_{22}$ has shown that the estimation of $\beta_{1}$ is sensitive to the choice of the prior values of $c_{22}$; hence, the use of an empirical estimate for the velocity variance $c_{22}$ is necessary.

6. Concluding remarks. This paper focused on modeling infant growth dynamics, mainly growth acceleration. We developed a Bayes methodology to assess the effect of exposure to a single EDC or mixtures of EDCs on growth dynamics. We have extended the previous B-SSVMs to enter exposure covariates in the acceleration model, which has never been studied in the literature. This extension provided a useful analytic toolbox to address various important questions arising in environmental health sciences while growth trajectories estimated from NGMs are competitive to previous methods for sparse data, such as a B-spline linear mixed effects model and a functional PCA.

Regularly-spaced longitudinal data are commonly collected in studies of infant's anthropometric measures because of study costs and limited manpower. This type of data often limits researchers to build dynamic statistical models for studying patterns of growth and development. However, this limitation may be alleviated by incorporating some reliable prior knowledge or relevant auxiliary data (e.g., growth curve data from WHO) into analyses, which has been shown in this paper to be advantageous in both bias reduction and statistical power gain for long-term growth dynamics. Indeed, we ran simulation studies to confirm the reliability for the detected associations of BMI growth acceleration with EDCs, and this confirmatory analysis was extended to compare with the SITAR model. Meanwhile, we were still cautious on statistical inference for some specific parameters (e.g., the variance of short-term growth dynamics) due to data sparsity.

Our contribution to the development of an efficient MCMC algorithm without data augmentation has made the needed computation substantially faster than the previous algorithm implemented by Zhu, Taylor and Song (2011), allowing researchers to perform data analysis with either the standard B-SSVM or NGM within a reasonable time. Our proposed algorithm directly drew posterior samples 
from posterior distributions, which enhanced fast mixing in posterior samples and increased significantly the computational efficiency. Moreover, the proposed algorithm enjoyed parallelization in computing to handle data with a large number of subjects. For practicality of our proposed method, an R package has been prepared and will be released to the public.

In this study, we discovered that the first trimester in utero exposure to BPA and phthalates was inversely associated with infant's growth acceleration. This inverse association indicates the delayed infant BMI peak and higher BMI (or fatty body mass) at the age of the first life milestone. It has been reported that such early growth deficiency with a warning sign of fatty body mass may have adverse health effects on infant's putative later outcomes (e.g., tempo of sexual maturation). Moreover, this delayed infant BMI peak could serve as a clinically important bio-marker concerning epigenetic changes. For instance, a recent study found that placental long interspersed nuclear element-1 (LINE1) methylation is negatively associated with prenatal phthalate exposure [Zhao et al. (2015)], and such changes in patterns of DNA methylation in the human placenta reliably and significantly result in alteration of infant growth [Banister et al. (2011)].

As an implication of the observational cohort design of our study, we were unable to make casual inferences about any association between the growth profile and in utero exposure to EDCs. Our effort has been centered on estimating effects of a single EDC or EDC mixtures on postnatal growth, by adjusting various confounding factors. Although this proposed methodology was illustrated on the ELEMENT cohort data, it may be applicable to the analysis of other similar growth cohort data.

This paper presents the first study that directly investigates infant's early life growth acceleration and its association with prenatal exposures to EDCs. The proposed Newton's growth model in this paper allowed us to estimate both individual acceleration and infant peak. These two estimated quantities, as well as others, are useful results to further understand many other relationships regarding early life growth characteristics with later life growth and development, and well-being.

Acknowledgements. The authors thank the participating mothers and children in the Early Life Exposures in Mexico to ENvironmental Toxicants (ELEMENT) study and thank the ELEMENT Principle Investigators for their insightful suggestions and constructive comments. We thank an Associate Editor and two referees for their critical review and valuable comments that led to a substantive improvement of this paper.

\section{SUPPLEMENTARY MATERIAL}

Supplement to "Bayesian analysis of infant's growth dynamics with in utero exposure to environmental toxicants" (DOI: 10.1214/18-AOAS1199SUPPA; .pdf). The supplementary document contains the details of the proposed MCMC algorithm and additional figures. 
R code for NGM (DOI: 10.1214/18-AOAS1199SUPPB; .zip). An MCMC algorithm written in R code for the NGM is publicly available.

\section{REFERENCES}

Afeiche, M., Peterson, K. E., Sanchez, B. N., Cantonwine, D., LamadridFigueroa, H., SchnaAs, L., Ettinger, A. S., Hernandez-Avila, M., Hu, H. and Tellez-Rojo, M. M. (2011). Prenatal lead exposure and weight of 0-to 5-year-old children in Mexico city. Environ. Health Perspect. 119 1436-1441.

AGARWAL, D. K. and GELFAND, A. E. (2005). Slice sampling for simulation based fitting of spatial data models. Stat. Comput. 15 61-69. MR2137218

BAEK, J., ZHU, B. and SONG, P. X. (2019). Supplement to "Bayesian analysis of infant's growth dynamics with in utero exposure to environmental toxicants." DOI:10.1214/18-AOAS1199SUPPA, DOI:10.1214/18-AOAS1199SUPPB.

Banister, C. E., Koestler, D. C., Maccani, M. A., Padbury, J. F., Houseman, E. A. and MARSIT, C. J. (2011). Infant growth restriction is associated with distinct patterns of DNA methylation in human placentas. Epigenetics 6 920-927.

Binkin, N. J., Yip, R., Fleshood, L. and TROwbridge, F. L. (1988). Birth weight and childhood growth. Pediatrics 82 828-834.

Botton, J., Heude, B., Maccario, J., Ducimetière, P., Charles, M. A., Basdevant, A., Borys, J. M., Bresson, J. L., Froguel, P., Lommez, A., Oppert, J. M. and Romon, M. (2008). Postnatal weight and height growth velocities at different ages between birth and $5 \mathrm{y}$ and body composition in adolescent boys and girls. Am. J. Clin. Nutr. 87 1760-1768.

Braun, J. M., Just, A. C., Williams, P. L., Smith, K. W., Calafat, A. M. and Hauser, R. (2014). Personal care product use and urinary phthalate metabolite and paraben concentrations during pregnancy among women from a fertility clinic. Journal of Exposure Science and Environmental Epidemiology 24 459-466.

Casals-Casas, C., Feige, J. N. and Desvergne, B. (2008). Interference of pollutants with PPARs: Endocrine disruption meets metabolism. Int. J. Obes. 32 S53-61.

Cole, T. J., Donaldson, M. D. C. and Ben-Shlomo, Y. (2010). SITAR-a useful instrument for growth curve analysis. Int. J. Epidemiol. 39 1558-1566.

Diamanti-Kandarakis, E., Bourguignon, J.-P., Giudice, L. C., Hauser, R., Prins, G. S., Soto, A. M., Zoeller, R. T. and Gore, A. C. (2009). Endocrine-disrupting chemicals: An endocrine society scientific statement. Endocr. Rev. 30 293-342.

Durbán, M., Harezlak, J., WAND, M. P. and CARroll, R. J. (2005). Simple fitting of subjectspecific curves for longitudinal data. Stat. Med. 24 1153-1167. MR2134571

González-Cossío, T., Peterson, K. E., Sanín, L. H., Fishbein, E., Palazuelos, E., Aro, A., Hernández-Avila, M. and Hu, H. (1997). Decrease in birth weight in relation to maternal bone-lead burden. Pediatrics 100 856-862.

Who Multicentre Growth Reference Study Group (2006). WHO Child Growth Standards: Length/Height-for-Age, Weight-for-Age, Weight-for-Length, Weight-for-Height and Body Mass Index-for-Age: Methods and Development. World Health Organization, Geneva.

JENNS, R. M. and BAYLEY, N. (1937). A mathematical method for studying growth in children. Hum. Neurobiol. 9 553-556.

Jensen, S. M., Ritz, C., Ejlerskov, K. T., Mølgaard, C. and Michaelsen, K. F. (2015). Infant BMI peak, breastfeeding, and body composition at age 3 y. Am. J. Clin. Nutr. 101 319-325.

Jones-Smith, J. C., Neufeld, L. M., Laraia, B., Ramakrishnan, U., GarciaGuerra, A. and Fernald, L. C. H. (2013). Early life growth trajectories and future risk for overweight. Nutr. Diabetes 3 e60. 
Kobrosly, R. W., Parlett, L. E., Stahlhut, R. W., Barrett, E. S. and Swan, S. H. (2012). Socioeconomic factors and phthalate metabolite concentrations among United States women of reproductive age. Environ. Res. 115 11-17.

LóPEZ-Pintado, S. and MCKeague, I. W. (2013). Recovering gradients from sparsely observed functional data. Biometrics 69 396-404. MR3071058

Marie, C., Vendittelli, F. and Sauvant-Rochat, M. P. (2015). Obstetrical outcomes and biomarkers to assess exposure to phthalates: A review. Environ. Int. 83 116-136.

Marsee, K., Woodruff, T. J., Axelrad, D. A., Calafat, A. M. and Swan, S. H. (2006). Estimated daily phthalate exposures in a population of mothers of male infants exhibiting reduced anogenital distance. Environ. Health Perspect. 114 805-809.

McKeague, I. W., Lopez-Pintado, S., Hallin, M. and Siman, M. (2011). Analyzing growth trajectories. Journal of Developmental Origins of Health and Disease 2 322-329.

NeAL, R. M. (2003). Slice sampling. Ann. Statist. 31 705-767. MR1994729

NHANES, I. (2009). Fourth national report on human exposure to environmental chemicals. In Department of Health and Human Services Centers for Disease Control and Prevention, Atlanta, GA.

Piaget, J. (2000). Piaget's theory of cognitive development. In Childhood Cognitive Development: The Essential Readings 33-47.

Preece, M. A. and BAines, M. J. (1978). A new family of mathematical models describing the human growth curve. Ann. Hum. Biol. 5 1-24.

RICE, J. A. and WU, C. O. (2001). Nonparametric mixed effects models for unequally sampled noisy curves. Biometrics 57 253-259. MR1833314

SAARNI, C. (1999). The Development of Emotional Competence. Guilford, New York.

Schettler, T. (2006). Human exposure to phthalates via consumer products. Int. J. Androl. 29 134-139.

Silverwood, R., De Stavola, B., Cole, T. and Leon, D. (2005). BMI peak in infancy as a predictor for later BMI in the uppsala family study. Int. J. Obes. 33 929-937.

Taylor, R. W., Grant, A. M., Goulding, A. and Williams, S. M. (2005). Early adiposity rebound: Review of papers linking this to subsequent obesity in children and adults. Curr. Opin. Clin. Nutr. Metab. Care 8 607-612.

Téllez-Rojo, M. M., Hernández-Avila, M., Lamadrid-Figueroa, H., Smith, D., Hernández-Cadena, L., Mercado, A., Aro, A., Schwartz, J. and Hu, H. (2004). Impact of bone lead and bone resorption on plasma and whole blood lead levels during pregnancy. Am. J. Epidemiol. 160 668-678.

WAHBA, G. (1978). Improper priors, spline smoothing and the problem of guarding against model errors in regression. J. Roy. Statist. Soc. Ser. B 40 364-372. MR0522220

YAO, F., MÜLLER, H.-G. and WANG, J.-L. (2005). Functional data analysis for sparse longitudinal data. J. Amer. Statist. Assoc. 100 577-590. MR2160561

Zhang, A., Hu, H., Sánchez, B. N., Ettinger, A. S., Park, S. K., Cantonwine, D., SchnaAs, L., Wright, R. O., LAMAdrid-FigueroA, H. and TEllez-Rojo, M. M. (2012). Association between prenatal lead exposure and blood pressure in children. Environ. Health Perspect. 120.

ZhaO, Y., Shi, H. J., Xie, C. M., Chen, J., Laue, H. and Zhang, Y. H. (2015). Prenatal phthalate exposure, infant growth, and global DNA methylation of human placenta. Environ. Mol. Mutagen. 56 286-292.

ZHU, B., TAYLOR, J. M. G. and SONG, P. X.-K. (2011). Semiparametric stochastic modeling of the rate function in longitudinal studies. J. Amer. Statist. Assoc. 106 1485-1495. MR2896851 


\section{J. BAEK}

DEVISION OF BIOSTATISTICS

AND Health SERVICES RESEARCH

DEPARTMENT OF POPULATION

AND Quantitative Health Sciences

University of Massachusetts Medical SCHOOL

368 Plantation STREET

WorCESTER, MASSACHUSETTS 01605

USA

E-MAIL: jonggyu.baek@umassmed.edu
B. ZHU

DIVISION OF CANCER EPIDEMIOLOGY AND GENETICS

NATiOnal CANCER Institute

NATIONAL Institutes of HEALTh

9069 Medical Center Drive, Room 7E618

BETHESADA, MARYLAND 20892

USA

E-MAIL: bin.zhu@nih.gov

P. X. K. SONG

Biostatistics DepartMENT

SChoOl of Public Health

UNIVERSITY OF MICHIGAN

1415 WASHINGTON HEIGHTS

ANN ARBor, Michigan 48105

USA

E-MAIL: pxsong@umich.edu

URL: http://www.umich.edu/ songlab 